\title{
Irrungen und Wirrungen in dem Kampfe gegen die Tuberkulose.
}

\author{
Von \\ Dr. H. F. Gerwiener, \\ zur Zeit loitender Arzt der Heilstatte.
}

Die Bezeichnung "Kriegstuberkulose" hat aus bekannten Gründen - wie so manche andere Einrichtung - im Laufe des Krieges ein vorïbergehendes Bürgerrecht erworben. Die ungeheuere Fülle der verschiedenartigsten tuberkulösen Erscheinungen an einem bis dahin nie gesehenen Untersuchungsmaterial hat in manchen Punkten auf Grund einwandfreier Beobachtungen und gesammelter Erfahrungen die gesamte Tuberkuloseforschung um ein Bedeutendes vorwärts gebracht, so dass wir bezüglich mancher wichtiger bis dahin noch ungeklärter Fragen ein beweiskräftiges Gesamturteil gewonnen haben.

So zeigt sich, dass manche Kapitel - ich nenne nur die besonderen Mitteilungen über endogene und exogene Reinfektion, traumatische Infektion, die diagnostische Verwertbarkeit des Tuberkulins usw. - als mehr oder weniger geklärt - vorläufig geringere Beachtung finden, während wieder andere allgemeinere liragen vor allem sozialhygienischer und sozialökonomischer Natur immer wieder durch neue Beobachtungen und Erfahrungen an der Hand verschiedener Auffassungen und Deutungen zur Diskussion herausfordern. Die grössten Fortschritte der bisherigen Tuberkuloseforschung verdanken wir eben dem jetzigen Kriege nur aus dem Grunde, weil er durch seine gewaltige Grösse und Ausdehnung gerade der Tuberkulose die ansgesprochene Gelegenheit bot, ihren weitgehendsten individuellen Eigensinn in jeder Form so recht zum Ausdruck zu bringen.

Die endogene Reinfektion als ausschliessliche Ursache der Tuberkulose der Kriegsteilnehmer heranzuziehen, ist sicher gewagt, obschon sio nach unseren Erfahrungen am wahrscheinlichsten ist. Die Gefahr einer massigen exogenen Reinfektion ist selbst unter den besten hygienischen Verhältnissen des Krieges zweifellos grösser als unter normalen Friedensverhältnissen, da die vielen unter den Truppen hefindlichen Bazillenspucker, insbesondere im Anfang des Krieges, 
ferner die durchseuchten Quartiere in Frankreich und Russland, und endlich die natürlich vermehrte Anzahl von Erkältungskrankheiten der Respirationsorgane dabei eine besondere Rolle spielen.

Auch die von von Hayeck (Wiener klin. Wochenschr. 1917, 1 und 2) angeführten Stützpunkte für die Annahme einer primären Erkrankung durch exogene massige Infektion: Fehlen einer familiären Belastung, eines tuberkuloseverdächtigen Habitus sowie tuberkuloseverdächtiger Symptome vor der manifesten Erkrankung, ferner der oft akute Beginn der Tuberkulose und vor allem ihr progredienter bösartiger Verlauf sind immerhin beweiskräftige Zeichen fehlender Immunität durch früher überstandene Infektionen. Auch von vielen anderen Seiten wurde der Kriegstuberkulose dieser bösartige und progrediente Verlauf als besonderes Charakteristikum zugeschrieben. Auf Grund anamnestischer Angaben und objektiver Befunde von über 1000 eigener Beobachtungsfälle möchte ich behaupten, dass sich der Verlauf der Kriegstuberkulose nicht wesentlich von dem der Friedenstuberkulose unterscheidet. In weitaus der grössten Mehrzahl der Fälle handelte es sich allerdings bei den schweren Formen meistens um dieselben Erscheinungen, wie sie von von Hayeck für die Annahme einer primären Infektion hervorgehoben wurden. Auffallend jedoch war - wie dies auch von $\mathrm{Hoch}$ ha us (Deutsche med. Wochenschr. 1916, 38) an der Hand desselben Beobachtungsmaterials nachgewiesen wurde, dass gerade von den schwersten Fällen über die Hälfte angab, früher nie lungenkrank gewesen zu sein - eine Tatsache, die auch bei der Feststellung der Tb.-Frage Schwerkranker immer wieder auffiel -. während die gelegentlichen Sektionsbefunde fast ohne Ausnahme bewiesen, dass jedesmal grössere, älte re Prozesse vorhanden waren, deren Entwickelung zweifellos längere Zeit vor dem Kriege lag.

Ausser dem vorwiegenden Fehlen einer erblichen Belastung und eines tuberkuloseverdächtigen Habitus zeigte eine nicht geringe Anzahl bei der Aufnahme sogar ein blühend gesundes Aussehen, das oft in wenigen Wochen bei pneumonieähnlichem Verlauf einer hochgradigen Kachexie Platz machte und zwar in auffallend vielen Fällen im Anschluss an eine akute Halsentzündung. Des weiteren dürfte es auch keineswegs als Zufall anzusehen sein, dass der grösste Prozentsatz dieser Fälle der ausgesprochenen ländlichen Bevölkerung angehörte.

Unter Berücksichtigung der gegebenen Momente bietet also der klinische Verlauf nichts Besonderes. Dass eben diese und auch andere Eigentümlichkeiten der Tuberkulose luäufiger beobachtet wurden, erklärt sich zum grössten Teil aus den hinlänglich bekannten Ursachen des grossen gleichartigen Materials, wobei die gleichen Altersstufen, die gleichgestellten Lebensbedingungen und andere gleichzeitige Einflüsse ihre bestimmte Rolle spielen. Hat doch auch die zufällige Anhäufung des Materials umgekehrt Fälle ausgedehnter Tuberkulose in ungewöhnlich grosser Anzahl zur Beobachtung gebracht, die mit den geringfügigsten physikalisch-klinischen Erscheinungen einhergingen, wie ich sie ebenfalls an demselben Material $z \mathfrak{u}$ beobachten Gelegenheit hatte und als besondere Eigentümlichkeiten seinerzeit aufgefasst und beschrieben habe (Beitr. z. Klin. d. Puberk., Bd. XXXV). Möglicherweise 
werden die genannten klinischen Eigentümlichkeiten einschliesslich der gesichteten Sektionsbefunde vielleicht unter besonderer Berücksichtigung und Beurteilung der städtischen und ländlichen Bevölkerung uns später bestimmte Anhaltspunkte an die Hand geben, um aus diesem Verhältnis und dem besonderen Verlauf der Tuberkulose erfahrungsmässige Schlüsse zu ziehen, auf die fortschreitende Abschwächung der Tuberkulose, Immunität sowie exogene und endogene Reinfektion.

Über andere natürliche Begleit- und Folgeerscheinungen des Krieges: Überanstrengungen, Entbehrungen, Erkältungs- und Infektionskrankheiten, Erschütterungen, Gasvergiftungen, Brustquetschungen und endlich Lungenverletzungen lautet das Urteil der meisten Autoren (Frieschbier, Gerhartz, Grau, Hochhaus, Orth, Moritz usw.) ziemlich übereinstimmend dahin, dass die genannten Ursachen nur in verhältnismässig wenigen Fällen mit Sicherheit für das Auftreten eirer primären Erkrankung verantwortlich $z u$ machen sind. Dass selbstverständlich die tuberkulös erkrankte oder auch in Heilung befindliche Lunge ungünstigeren Verhältnissen ausgesetzt ist, bedarf keiner besonderen Erwähnung. Bei der gewiss nicht geringen Anzahl sämtlicher Lungenverletzungen und der noch bei weitem viel grösseren Anzahl latent tuberkulöser Soldaten muss man sich allerdings wundern, dass nur in verhältnismässig wenigen, Fällen die Reaktivierung einer Lungentuberkulose im Anschluss an Brustverletzungen festgestellt worden ist; "denn die Erfahrungen, die man in diesem Kriege bei Lungenschüssen gemacht hat, sprechen dagegen; hat man doch Heilung der durchschossenen Lunge eintreten sehen, obwohl die andere tuberkulös erkrankt war". (Orth, Zeitschr. f. Tuberk. 2ら́, V.)

Öfter - wie dies auch von französischer Seite behauptet wird sah man das Auftreten tuberkulöser Prozesse im Anschluss an Lungensteckschüsse und zwar nicht selten in der Nähe des eingeheilten Geschossstückes. Eine Anlage für Tuberkulose nach Schussverletzungen kerngesunder Lungen wird nach den bisherigen Erfahrungen nicht angenommen.

Eine direkte Infektion der Lunge durch das Geschoss selbst würde gewiss zu den Seltenheiten gehören, wenn es überhaupt möglich ist, für diese Tatsache einwandfreie Beweise zu erbringen. Immerhin dürfte es zu den Zufälligkeiten gehören, dass ein Geschoss eine frischkavernöse Lunge eines Soldaten durchschlägt und als Durch-oder Steckschuss die gesunde Lunge des hinter ihm stehenden trifft. Handelt es sich um ein zerklüftetes Geschoss, etwa einen Granat-, Holz- oder Steinsplitter, so ist die Infektion nicht nur möglich, sondern meines Erachtens sehr wahrscheinlich. Nimmt man weiter hinzu die verhältnismässig grosse Anzahl teils kriegsfreiwilliger, teils nach oberflächlicher Untersuchung eingestellter Schwerkranker, wie sie im Antang des Krieges oft in grosser Anzahl von der Front in die Heimatlazarette zurückgeschickt wurden, oder vergegenwärtigt man sich die unter denselben Umständen gegebenen Verhältnisse bei der gewiss stärker tuberkulös durchseuchten französischen Armee, so glaube ich, dass man unter einigermassen "günstigen" Bedingüngen eine direkte Infektion nicht einfach von der Hand weisen kann, um so mehr, da andere zum Teil unwahrscheinlichere Zufälligkeiten in 
den Reihen der Millionenheere als überlieferte 'Tatsachen keine grossen Seltenheiten sind. Soweit man auch im besonderen über die soeben kurz erwähnten 'Tatsachen ein bestimmtes Urteil zu fällen berechtigt ist, so wird dennoch keiner leugnen dürfen, dass uns nach Kriegsschluss das Gesamtergebnis dennoch manche Überraschung bringen wird.

Vielleicht - und dies möchte ich sowobl mit Rücksicht auf die erwähnten 'l'atsachen und an der Hand der folgenden Ausführungen mit ziemlicher Sicherheit behaupten - wird uns gerade in der entscheidendsten und brennendsten Frage der Tuberkuloseforschung, nämlich bezüglich der Ausdehnung der Tuberkulose erst nach dem Kriege die grösste und hoffentlich gewiss nicht unerfreulichste Überraschung zuteil werden.

Wie wertroll und unvergänglich auch in wissenschaftlicher Beziehung die hervorragenden diagnostischen Fortschritte in der Tuberkulose-Erkenntnis sind, vor allem unter Hinzuziehung der serologischen Untersuchungsmethoden, so werden sie dennoch letiten Endes in der jetzigen Anwendungsweise nicht den Erwartungen entsprechen, die man in der Hauptaufgabe, der Tuberkulosebekämpfung nach dem Kriege, an sie stellen wird.

Gerade die verfeinerten und subtilsten Cntersuchungsmethoden, die auf Grund scharf beobachteter Reaktionen in der Hand des erfahrenen und geübten Praktikers und Klinikers schon so manche krfolge gezeitigt haben, sie werden in der Hand des L'ngeübten nicht den erwünschten Nut\%en bringen, in manchen Fällen sogar nicht unerheblichen Schaden anrichten. Gegenüber der gewaltigen Ausdehnung der Tuberkulose wird erst die allgemein erworbene sicliere Beurteilung ausschlaggebender Erscheinungen den praktischen Wert - und darauf kommt es in erster linie an - erkennen lassen, während wir nach den bisherigen Erfahrungen nur zu oft davon überzeugt werden, in welch ausgedehnter Weise die statistischen Aufzeichnungen über die Ausdehnung der 'luberkulose durch falsche Beurteilung beeinflusst sind. Nur die absolute Sicherheit in der Anwendung und Deutung unserer bekannten Untersuchungsmethoden zur Feststellung einer T'uberkulose wird uns davor bewahren, dass wir nach dem Kriege statistischen Zahlengrössen gegenüberstehen, denen unsere bisher ausreichenden und mit Erfolg angewandten Bekämpfungsmassnahmen in keiner Weise gewachsen sind.

Es besteht kein Zweifel, dass die Ausdehnung der Tuberkulose infolge der Eigentümlichkeiten des Krieges eine natürlich grössere geworden ist. Fs ist für den genauen Beobachter aber auch keine Frage, dass die augenblicklichen, zum 'Teil auch nur mutmasslichen Zahlenverbältnisse über die Ausdehnung der Tuberkulose gewaltig übertrieben sind, wenigstens bei der gesamten männlichen Bevölkerung, soweit dieselbe für militärdienstliche Verhältnisse überhaupt und besonders rorübergehend in Frage kommt. Ton vielen Autoren wurde bereits diese auffallende Erscheinung mit der gleichgrossen Anzahl erwiesener Fehldiagnosen in Zusammenhang gebracht und erörtert. Wenn es auch vielleicht etwas $z u$ weit gegangen ist, wenn Fränkel (Mïnch. med. Wochenschr. 1916, 1109) die Zahl der Fehldiagnosen 
mit $60 \%$, Blümel (Mediz. Klin. 1915, 884) sogar mit 80\% angegeben haben, so stimmen doch im allgemeinen die Ansichten namhafter Autoren darin überein, dass unter der Diagnose Tuberkulose eine sehr erhebliche Anzahl Neurastheniker, Bronchitiker und nicht zuletzt Simulanten marschieren, wie dies auch besonders im Anfang des Krieges aus den interessanten Berichten der militärischen Beobachtungsstationen hervorging. So gab auch de la Camp (Mediz. Klinik 1916, 18) ungefähr ein Drittel seines Beobachtungsmaterials als nicht tuberkulös an.

Bei der anerkannten Schwierigkeit der Diagnosestellung und nicht zuletzt infolge der gewaltigen Überlastung der Militärärzte waren diese oft genug gezwungen, sich auf die häutig übertriebenen Angaben der Kranken allein zu verlassen.

Unter noch grösseren Schwierigkeiten batten in dieser Beziehung die Frontärzte, insbesondere die kaum Ausgebildeten, zu kämpfen, und selbst die nicht immer fachärztlich geleiteten Beobachtungsstationen und Lazarette verliess mancher $D$. U.entlassene "Lungenkranke", der es mit oder auch ohne eigene Schuld verstanden hatte, auf Grund seiner Beschwerden den Arzt zu täuschen. Dass diese Zahl allein keine geringe war, konnte ich während fast einer 3 jährigen militärärztlichen Beobachtungstätigkeit oft genug bei den wiederholten Aufnahmen auf unserer Beobachtungsstation feststellen. Abgesehen von den vielen Komplikationen der Magen-Darmerkrankungen, der Neurosen, Psychosen und der nicht spezifischen Erkrankungen der Respirationsorgane, die alle mehr oder weniger als begleitende Komplikationsmomente angesehen und gedeutet worden waren, ergab auch die grosse Menge der ausgesprochenen Simulanten oft recht bedenkliche Zahlen, die auf Grund einer eingehenden Anamnese, zum Teil mit Hilfe der Familie und zuständigen Ortsbehörde des Erkrankten, sowie nach genauester Untersuchung und Beobachtung einwandfrei festgestellt worden waren.

Es würde zu weit führen, die bekannten und häufig beobachteten 'Täuschungsversuche dieser Art, die bei einem Beobachtungsmaterial von über 1000 Fällen gemacht wurden. mitzuteilen. Nur einen interessanten charakteristischen Fall ron Simulation möchte ich kurz. mitteilen, weil er auch mit Rücksicht auf die gestellte Diagnose eine besondere Bedeutung hat, auf die ich nachher zurückkomme.

Es handelt sich uin einen 40 jährigen Mann, der wegen ausgedebnter, offener, fieberhafter Lungentuberkulose sowie Kehlkopf-, Darm- und Blasentuberkulose zwecks Einleitnng des D. U.-Verfahrens zu uns abgeschoben wurde, nachdem er bis dahin in einem anderen Lazarett ungefähr 9 Monate (!) lang beobachtet und behandelt worden war.

Wegen der foudroyanten ständigen Blutungen aus Lunge, Darm und Blase wurde erst nach 5 Tagen eine eingehendere Untersuchung vorgenommen und diese auch nur aus dem Grunde, weil die rektale Fieberkontrolle die orsteu bedenklichen $\mathrm{Z}$ weifel in der Echtheit des gesamten unsicheren klinischen Bildes bestätigte. Die Untersuchung sämtlicher Organe, aus denen die ungewöhnlichen Blutmengen stammen sollten, ergab nicht den geringsten Anhaltspunkt für eine T'uberkulose, die auch durch wiederholte physikalische und röntgenologische Untersuchungen nicht festgestellt werden konnte.

Da der Mann im Nebenberuf Zauberkunstler war, konnte unsere weniger begabte Findigkeit den artefiziellen Ursprung der grossen Blutmengen nicht entdecken. Vielleicht würde dennoch dieses, zauberhafte“ seltene Krankheitshild bei 
uns eine schnellere natïliche Erklărung gefunden haben, wenn nicht die ,nounmonatige Missgeburt" des beigegebenen Krankenblattes den meh rmsligen Nachweis schwerster pathologischer Veränderungen, insbesondere über den Lungen, und den Massenbefund von Tuberkelbazillen enthalten hätte.

Der angeführte Fall ist wohl das krasseste Beispiel dieser Art und lässt sowohl auf die Verwertbarkeit mancher Krankenberichte wie auch auf die Ausdehnung der Simulation berechtigte Schlussfolgerungen $z u$, die uns auch später noch oft genug bei der Durchsicht anderer Krankenblätter in allerdings nicht so aufiallender Weise bestätigt wurden. Jedenfalls aber - und das wurde auch von anderen Beobachtunisstationen mehrfach hervorgehoben - werden derartige ärztliche lookumente, auch in milderer Form, der nächsten Beobachtungsstelle die richtige Diagnosestellung bedeutend erschweren, of sogar unmöglich machen, mit Ausnabme all der anderen Fälle, die bereits schon vorher auf Grund ähnlicher Befunde wegen "Tuberkulose" als dauernd D. I., zum Teil noch als Rentenempfänger entlassen wurden.

Die letzte Kontrolle derartiger 'Tatsachen haben nicht selten die lungenheilstätten, in denen dann diese "Kriegstuberkulösen" oft genug kurz vor der erneuten militärärztlichen Untersuchung Aufnahnse. gefunden haben.

Die spätere statistische \%usammenstellung dieses gesichteten Heilstättenmaterials wird gewiss in mancher Beziehung den übergrossen l'essimismus über die Auslehnung der Tuberliulose in anderem İichte elscheinen lassen.

Es ist nicht schwer, besonders unter den derzeitigen Verhältnissen die Ausdehnung unserer grössten Volksseuche mit den verschiedenartigsten Momenten in ursächlichen Zusammenhang zu bringen. Unter diesem sicher vorhandenen, nicht geringen Einfluss ist es in mancher Hinsicht leichter geworden, viele tuberkuloseverdächtige Erscheinungen in diesem. Sinne verantwortlich zu machen und nicht zum wenigsten wird diesen Momenten eine ausschlaggebende Bedeutung zukommen, wenn die Zuverlässigkeit der diagnostischen Hilfsmittel oder auch die praktische Ubung des Untersuchers selbst vieles zu wiunschen ïhrig lässt.

Da wir auch nach dem Kriege in Ermangelung einer Anzeigepflicht für tuberkulös Erkrankte nur auf die statistischen Angaben der Militärbehörden. der Lungenheilstätten und Fürsorgestellen angewiesen sein werden, so ist es ohne weiteres klar, dass diese Angaben bezüglich ihrer grösstmöglichen Genauigkeit ganz besondere Aufmerksamkeit beanspruchen, wenn wir uns nach dem Kriege über die Ausdehnung der 'I'uberkulose auch nur ein annähernd richtiges Jild machen wollen.

Auf Grund mehrerer Beobachtungen möchte ich hier nur einige lunkte herausgreifen, die bezüglich der statistischen Angaben für die Heilstätten in Frage kommen. Die Behauptung von Meyer (Ref. Zeitschr. f. Tuberk., Bd. 26), dass im Frieden die Mehrzahl der Heilstätteninsassen nicht tuberkulös gewesen sei, und dass es sogar schwer gehalten habe, wirklich Lungenkranke in Heilstätten unterzubringen, ist sicher übertrieben: wohl aber lassen die gemässigteren Mitteilungen 
erfahrener Heilstättenärzte vermuten, dass man dieser Erkenntnis mit einer verständlichen zweideutigen Weitherzigkeit schon lange gegenüberstand. Die militärärztliche Sichtung fast unserer gesamten männlichen Bevölkerung nun hat auf die Zusammensetzung des Heilstättenmaterials in dieser Beziebung einen natürlichen günstigen Einfluss gehabt, dennoch aber auch wieder neue charakteristische Erscheinungen hervorgerufen, von denen einige sofort in die Augen springen. Die Durchsicht einer entsprechenden Anzahl alter Krankenberichte bestätigt die alltägliche Wahrnehmung, dass einerseits die Anzahl der ausgesprochen schweren Fälle von Tuberkulose und andererseits die Menge der leichtesten bzw. nur verdächtigen "Lungenspitzenkatarrhe" in gleichem Verhältnis zugenommen hat. Ohne auf die erstere leicht erklärbare Erscheinung näher einzugehen, verdient die zweite und bei weitem wichtigere l'atsache, bei dem jetzigen durch den grossen Andrang zu den Lungenheilstätten hervorgerufenen Platzmangel unsere grösste und erneute Aufmerksamkeit. Wenn jemals, so muss besonders jetzt bei unserer Heilstättenauswahl oberster Grundsatz werden, dass nur wirklich geeigneten Fällen eine Aufnahme gewährt wird. Ohne Frage kommen hierfür - wie jeder Heilstättenantrag deutlich hervorhebt - , nur diejenigen Kranken in engere Wahl, bei denen voraussichtlich eine wesentliche Besserung oder Heilung zu erwarten ist. Die erste Entscheidung, dieser verantwortungsvollen Auswahl liegt zunächst und oft ausschliesslich in der Hand des praktischen Arztes. Ihr folgt in vielen Fällen die vertrauensärztliche definitive Begutachtung eines zweiten Arztes (Kreisarzt), oder endlich die Entscheidung auf Grund einer längeren Beobachtung in einem Krankenhaus (Vorund Durchgangsstationen).

Wenn schon an und für sich die sichere Diagnose einer initialen tuberkulösen Lungenerkrınkung zu den grössten Schwierigkeiten für den Arzt gehört, so glaube ich, wird es in vielen Fällen für ihn eine Unmöglichkeit bedeuten, wenn er diese Entscheidung an der Hand diagnostischer Untersuchungsmethoden treffen soll, für die er nicht immer die ausreichende Übung und Erfahrung besitzt. Noch verantwortungsvoller, vielleicht auch noch schwieriger, wird für ihn dann die weitere Entscheidung sein, $a b$ es sich in dem gegebenen Falle um eine behandlungsbedürftige, $d$. h. aktive Lungentuberkulose handelt. Diese Frage ist während des Krieges aus dem Grunde als eine der brennendsten zu betrachten, weil sie in Anbetracht der entgegengesetzt laufenden Zahlenverhältnisse über Heilstättenanträge und Bettenanzahl in erster Linie der gesunden Lösung bedarf. Diese Möglichkeit ist aber nur dadurch gegeben, dass die Auswahl für die Heilstätten nur solchen Stellen überantwortet wird, die den vermehrten Aufgaben auch gewachsen sind. Bei der schon betonten Schwierigkeit der Diagnosestellung, erschwert durch die stets verfeinerten und dabei zum Teil noch unsicheren diagnostischen Hilfsmittel, ist es meines Erachtens eine Ungerechtigkeit, von dem überlasteten praktischen Arzte ein fachärztliches Urteil auf einem Gebiete zu verlangen, auf dem selbst erfahrene Spezialisten noch uneinig sind und das in sozialökonomischer und sozialhygienischer Beziehung bei uns den ersten Platz einnehmen soll. Um so mehr muss es da gerade mit Rücksicht 
auf die hervorragenden fortschrittlichen Bestrebungen in der Tuberkuloseforschung der letzten Jahre auffallend erscheinen, wenn recht oft die gutachtlichen Untersuchungsbefunde, und vor allem die Ansichten der praktischen Ärzte, über die Notwendigkeit der Aufnahme in einer Lungenheilstätte Mängel erkennen lassen, die mit den wissenschaftlichen Fortschritten und besonders mit der wachsenden Bedentung der I'uberkulosebekämpfung nur schwer in Einklang $z u$ bringen sind. Zur Erhärtung meiner Behauptung möchte ich an der Hand der verschiedensten Untersuchungsbefunde, von denen ich wegen Raummangels an dieser Stelle nur wenige im Original wiedergeben kann, nur die auffallendsten Ungleichheiten und Widersprüche gegenüber unseren Aufnahmebefunden hervorheben, in der Hauptsache aber sogenannte diagnostische Unmöglichkeiten näher beleuchten, die mit ziemlicher Sicherheit die Kenntnis selbst der physikalischen Lungenuntersuchung bezweifeln lassen und mit Rücksicht anf die gemachten Schlussfolgerungen bezüglich der Bewertung und Deutung der Untersuchungsergebnisse unbedingt den ungeiibten oder stark beeinflussten Untersucher erkennen lassen.

1. A. R., 37 Jabre. Paralytischer Thorax bis 3 oben (?). Über der Lunge links verschärftes Atmungsgeräusch; Exspirium verlängert. Kein Fieber mehr, kein Durchfall; keine Blutungen mehr, seit zirka finem Monat.

Diagnose: I,angentuberkulose Stadium 11 (!).

Der Patient starb 6 Wochen nach der Aufnahme an einer ausgedehinten kavernösen Lungentuberkulose.

2. W. Sch., 36 Jahre. Linke Spitze Schallverkurzung. Über der linken Spitze bronchial-klingendes (!) Atmungsgeräusch, verlängertes Exspirium. Rechte Spitze raulses Inspirium, abgeschwathtes Exspirium.

Diagnose: Lungenspitzenkatarrh (?).

És handelte sich um eine doppelseitige, geringfïgigste, alte, abgeheilte Lungenspitzentuberkulose. Der Untersuchungsbelund sollte nur in der \%usammenstellung ron ,Schallverkürzung - bronchialklingendes Atmungsgeräusch", "Lungenspitzenkatarrh" als ein bekanntes und oft wiederkehrendes Charakteristikum hervorgehoben werden.

3. J. K., 56 Jahre. K. o. v. und hinten Schallverkürzung, sonst voller Klopfschall. K. v. o. zeitweise kleinblasige klingende (!) Gerdiusche. Das Atmungsgeräusch ist verstärkt (!).

Diagnose: Rechtsseitiger Langenspitzenkatarrh.

$D_{\text {a }}$ es sich um einen alten abgeheilten Fall handelte, der keine kleinblasige klingende Geräusche mehr aufwies, wurde Patient bereits nach 14 Tagen als gesund und voll erwerbsfähig entlassen.

4. I. T., 26 Jahre. Abgeschwächtes Atmen uber der rechten Spitze (R. supraclavicular). Links normale Verhältnisse (!). R. supra-clavicular undeutliches (?) Atmen mit knirschenden (!) Geräuschen. Giemende Geräusche auch (?), vereinzelt aber der rechten (?) Lunge

Diagnose: Tuberkulöser Lungenkatarih (!).

Der betreffende Patient wurde 2 Tage nach der Aufnahme, nachdem er sich von der Reise etwas erholt hatte, wegen seines desolaten Zustandes (beiderseits progressives III. Stadium) dem Krankenhaus überwiesen. 
5. W. B., 15 Jahre. Zwischeu beiden Schulterblattern verscharftes Atmon and Knisterrayseln; in der (?) Schlüs-eltueingrubo Atmung etwas brouchial (l); starke (!) Kasselgaräusche vorne (?) wairnelimbar.

Diagnose: Phthisis pulmodaris. Chrunische Bronchitis.

Unsere Diagnose lautete auf eine geringfügige, in Abheilung befindliche Lungenspilzentuberkulose.

6. J. Sch, 56. Jahre. Daimpfung des linken Oberlappens.

Diagnose: Lungentuberkulose.

Der Befund erscheint etwas dürftig und wenig beweisend für Lungentuberkulose. Wir stellten die Diagnose: Alte diffusse Bronchitis. Emphysem.

7. H. B., 23 Jahre. Die Lungenspitzen sind angegriffen (!) meist links; feinblasigra Rasseln uber der ganzen (!) l,unge.

Dingnose: Beginnende Lungentuberkulose.

Es handelte sich nach unserem Befunde um eine alte, diffusso Bronchitis.

8. J. P., 35 Jahre. Klopfschall ist hinten links uber dem Mittel- (?) und Unte l-pprn abgeschwächt bis (?) zum Schulterblatt. Atmurgsgeräusch über der Däıpfuıg (9) verschärft mit spärlichen Kasselg-1äuschen dirchsetzt.

Diagnose: Leiciıter Lungenkatarrh ohne Komplikationen (!).

Der Fall wurile nach 25 lagen als ungetiunet wegen progressiver fieberhafier Lungentuberkulose dem Krankenliause üherwiesen.

9 H. Sch., 27 Jalire. Schallverküzung vorne beiderseits bis 2 . Kosta. R. h. bis halb huks binten bis ${ }^{1}{ }_{3}$ Skapula. K. v. o üher der Spitze (!) rallh, unhestimmt mit ventängrrtem. fast handiend.n Ex-p 1 i.m; abwarts (!) vesiko-bıonchial bzw. (.) Ver-charft vesskulär, in der soits Gurren.

[.. v. ve-ikolirouchial-veldangertes Ex pirium, raub, in der Seito verschürft, uber Spize abges bwäclit. L. h. o. abyenchwïelit b-a ', Skapula, verschwommen (!) uniein, aınälsermi unhesıimmt (!) im verläıgerten Exspirium. R. b. o. entsprech.rad r. $\nabla$. bis $1 / 2$ Sk pu'a Olinn elnige Unreluhoiten.

Diagnuse: Luagentuborkulose fast Il. Stadiun.

'Irotz. des „äusserst genauen" und komplizierten Befundes. in dem auch weniger bekanute Anskuit.tionsbezeichnungen zu Wort kommen, konnte der Jatient nach Abheilung seiner Bronchitis nach 5 Titgen entlassen werden.

10 L. B., 28 Jahre. R. v. o. bis 2 . Kosta, hinten bis Schulterblattgräte ab. yekturzter Schall, ebrnso l. v. o. Lis Schlüsseibein und hinten uber der spitce. Daselbst verlänzeites haurheniles Exspirium. Rechts einize tockene Rasselgeräusche. Aliends Temperatursteigerangen! Wiederholte (!) Blutungen, die letzte vor 8 Tagen.

Diatonose: Chronische (!) Lungentuberkulose 2. Grades, veraltet (?).

Es hanitelte sich um eine doppel-eitige aktive Lungentuberkulose, für die allerdings mehr die obige Aufiassung über Aktivität, als die Form der l'uberkulose, "veraltet" erscheint.

11. J. H, 28 Jahre. R. v. bis 4. Kosta (!) D\&mpfung. R. v. abgeschwächtes Atmen bis 4 . Kippe. Exspirıum vir-cnäıft: hier (?) hört man einzelues Knacken.

Diagnuse: Lungenspizznkatarrh zechts (bis 4. Rippe) (l). Beginnender Lungeuspitzenkatarih links (?).

Belund und liarnose sind in ihrem Zusammenhang, besonders links, wenig rerständlich. Nach unserem Befunde bestand eine aktive Lungen- und" Kehlkopftuberkulose.

12. F. S., 40 Jahre. R. $\nabla$. o. Schallverkürzung bis 2. J. C. R. Über dieser Partie bestelit verlangertes und verscharftes Ex-pirium. keine Ra-s lger.usche (!) Sonstige Lunge ohne patbologische Veländerungen. Tuberkulinreakıion pusitiv. 
Nach dem okigen Befunde ist positive Tuberkulinreaktion nicht erklärlich. 'lemperaturangabe fehlt. Bei dem Falle konnten hier .. ebenfalls" keine sicheren Anhaltspunkte für eine behandlungsdïrftige Lungentuberkulose festgestellt werden.

13. E. Sch., 39 Jahre. Perkutorisch über beiden Lungenspitzen 1!) Dämpfung; links mit tympanitischem Beiklang.

Auskultation: I. v. o. broneho-amphore (! Atmung mit vereinzelten trockenen bis (!) mittelblasigen Rhonchi. L. h. o. his Schulterblattmitte bronchiales Atmen mit mittleren und gröberen Rbonchi. Allenthalben (!) broncho vesikulär's Atmen nit Rhonchi, besonders 1. v. u.

Uber der rechten Seite v. o. scharfes Bronchialatmen mit mittleren und grossblasigen Rasselgeräuschen, die fast klingend sind. R. v. (?) ganz scharfe Atmung mit mitjeren Rhonchi. R. h. bis Schulterblattspitze Bronchialatmen mit scharfen mittleren und gröberen Rhonchi.

Patient ist wegen der Schwere der Erkrankun (Ill. Stadiüm) zur Heilstittenkur ungeeignet.

Trotzdem wurde der latient - wenn auch erst nach 4 Jahren auf Grund eines anderen Gutachtens milderer Form hier aufgenommeri. Es handelte sich um eine gutartige, alte fibröse Lungenspitzentuberliulose ersten Grades. Auf manche Iinkorrektheiten. zum Teil [rumöglichkeiten besonder's hinzuweisen, eriibrigt sich.

Der obige Befund ist der einzige von den angeführten Fälen. der nicht als lezte gutachtliche Iusserung die Aufnahme in unsere Heilstätte bestimmte. Fr liegt 4 .Jabre zuriick. Schon aus diesem Grunde dürte dieses anskulatorische - Knriosum" einige Beachtung beanspruchen.

Hiermit möchte ich die kleine Bliitenlese schliessen. Es würde zu weit führen. ausserdem noch die vielen anderen lintersuchungshefunde mitzuteilen, die keinen Anspruch auf wissenschaftliche Wertung verdienen. Des weiteren sollen auch nicht die vielen minimalen Befunde mitgeteilt werden, die keine Inhaltspunkte für eine tuberku!öse Frkrankung enthalten, auf Grund deren aber immer die dringende Notwendigkeit eines Heilverfahrens leichtfertig beantragt wird, oft genug mit der unerklärlichen Begründung, dass mit dem Heilverfahren durchaus nicht mehr gewartet werden könne. Ohne auch weiter in demselben Sinne die oft tragliche Motivierung in der gutachtlichen Beantwortung der Fragen über die vorliegende Berutsunfähigkeit näher zu erörtern, erscheint es in Anbetracht nancher Befunde of auch recht bedenklich, wenn eine andere wichtige Frage des Gutachtens, nämlich die. ob zur Sicherstellung der Diagnose und l'rognose eine kume Beobachtung in einem Krankenhause notwendig oder zweckdienlich sei, in fast allen Fälen mit einem iberzeugten ..Nein " beantwortet wird. Und was endlich eine andere Frage: ... Besteht Verdacht auf Vorspiegelung oder libertreibung"? angeht, so findet sich diese ausnahmslos mit einem kurzen "Nein" erledigt. Mit Rïcksicht auf die obigen Beispiele darf mit Recht die Stichhaltiglieit dieser kurzen Antworten oft bezweifelt werden. Die ausserordentliche Wichtigkeit derselben für die weitere Begutachtung und Behandlung des Kranken bedarf keiner besonderen Erwähnung.

Was die Befunde selbst betrifft, so kann man fast durchweg feststellen, rass zumeist $z$ u viel diagnostiziert wird, eine Tatsache, die 
bekanntlich den meisten ungeübten und unertabrenen Unfersuchern eigen ist, die aber auch durch manche andere Finflisse subjektiverer Natur eine natürliche Erklärung findet. Im grossen und ganzen jedoch kann man sich oft nicht des Eindrucks erwehren, als seien die wissenschaftlichen Bezeichnungen bei einer Lungenuntersnchung noch vage unsichere Begriffe.

Wenn auch vielleicht bei der Diagnosestellung keiner anderen Erkrankung das subjektive Emptinden eine so grosse Rolle zu spielen pflegt, wie bei dem ausgedehnten Symptomenkomplex der Lungentuherkulose, so muss demgegenüber auf das Entschiedenste betont werden, dass in Anbetracht der weittragendell Folgen hier in erster Linie die wissenschaftlichen Begriffe fïr die pathologisch-anatomischen Verhältnisse an die bestimmtesten und klarsten Formen gebunden sein mïssen, und lass es nicht nur wünschenswert erscheint. wie Goldscheider (Deutsche med. Wochenschr. 1918, 4) hervorhebt. sondern für die Praxis zu einer dringenden Notwendigkeit geworden ist, dass über gewisse grundsätzliche Fragen der Tuberkulosediagnostik eine Finigung erzielt wird. Es ist von weitaus grösserer liedeutung, bei einem Patienten mit Sicherheit über den Lungenspitzen Rasselgeräusche oder Bronchialatmen festgestellt zu haben, als mit derselben Sicherheit bei einem anderen Patienten ein systolisches Herzgeräusch, eine Leber- oder Milzsergrösserung. Letztere Erscheinungen lassen die verschiedenartigsten Deutungen zu, während im ersten Falle mit wenigen Ausnahmen die inhaltschwerste Diagnose gestellt ist. Und nehmen wir in beiden Fällen das Gegenteii, d. h. eine Fehldiagnose an. so sind doch im ersten Falle die Folgen bei weitem schwerwiegender urd nachteiliger, wie bei jeder anderen chronischen Erkrankung. Und die Summe all dieser genannten Schwierigkeiten und Unsicherheiten ist gewöhnlich die Verlegenheitsdiagnose (B andeli el' und Röke) "Lungenspitzenkatarrh", eine Bereichnung, die allerdings im Laute der Jahre ihre ungekündigte Stellung behauptet hat, die aber vielleicht nur in ihrer therapeutischen Anwendung manchnal eine gewisse liedeutung besitzt. Wenn wir an der Hand unserer Befunde sehen, zu welchem wissenschaftlichen Missbrauch man hierbei in diagnostischer Bexiehung gekommen ist, so braucht man sich nicht mehr über die unklaren Vorstellungen zu wundern, die bëi Patienten und uberhaupt in Laienkreisen unter der Bezeichnung "Lungenspitzenkatarrh" dadurch hervorgerufen werden. Wiederholte Nachfragen hei meinen Patienten bestätigten immer wieder die bekannte Äusserung, dass ihr "Lungenspitzenkatarrh" mit Tuberkulose nichts zu tun habe, und dass dieser nur eine leichte vorübergehende Erkältung sei. Die Bedeutung dieser wichtigen volkserzieherischen Frage bei der Tuberkulosebekämpfung soll hier nicht weiter erörtert werden.

Noch ein Wort über einige andere Entgleisungen der angeführten Untersuchungsbefunde.

Zunächst erscheint es erstaunlich, in welch grossem Masse und mit welcher Leichtigkeit Bronchialatmen diagnostiziert und bewertet wird. Abresehen von seinem an einzelnen Stellen physiologischanatomisch bedingten Auftreten weist Bronchialatmen durchweg auf 
eine Solidifikation in der Lunge hin und meistens auf-solche pathologische Veränderungen in derselben, die für gewöhnlich eine erhebliche Dämpfung voraussetzen und dann wohl über das I. Stadium hinausgehen, geschweige denn die Bezeichnung „Lungenspitzenkatarrh" verdienen. In unseren Fällen also, wo dasselbe als spezifischer Ausdruck einer tuberkulösen Lungenerkrankung gebraucht wird, kann nur die Annahme gestattet werden, dass die Auffassung und Deutung des Begriffs Bronchialatmen eine falsche ist.

Ferner gibt es auch für die Bezeichnungen: Kleinblasige-klingende Geräusche, verstärktes Atmungsgeräusch, undeutliches Atmen, raubes verschärftes Atmen, Dämpfung uber dem „linken“ Mittellappen usw. keine andere Erklärung als vielleicht die der grössten Oberflächlichkeit bei der Untersuchung; denn in vielen - nicht allen - Fällen liegt ja bekanntlich der Hauptgrund der Fehldiagnose weniger in der Unkenntnis des Untersuchers, als vielmehr in der mangelhaften und oberflichlichen Untersnchung selbst. Dass diese aber gerade bei der verantwortungsvollen Diagnosestellung der Lungentuberkulose die aller. geringste Entschuldigung finden kann, liegt nach den gemachten traurigen Erfahrungen und mit Rücksicht auf die bevorstehenden Aufgaben in der Tuberkulosebekämpfung klar auf der Hand.

Dass wir auch in der Auffassung über eine weitere entscheidende Frage, nämlich der Aktivität der tuberkulösen Erkrankung bzw. der Behandlungsbedürftigkeit derselben in unseren Befiunden auf die grössten Inkonsequenzen stossen, kann insofern kein Wunder nehmen, weil in der Beantwortung dieser zur Zeit brennendsten Frage die Anschauungen überhaupt noch recht verschieden sind und der dringend notwendigen Klärung bedürfen.

Die vermehrten diagnostischen Schwierigkeiten werden jetzt und besonders nach dem Kriege nicht in erster Linie darin bestehen, mit "mathematischer Sicherheit" eine stattgehabte tuberkulöse Infektion überhaupt festzustellen, sondern zu entscheiden, ob es sich um eine aktive Tuberkulose, d. h. eine behandlungsbedürftige Erkrankung handelt. Nur in dieser richtigen Beurteilung liegt ohne weiteres die Entscheidung, ob sofort die entsprechende Behandlung des Lungenkranken - in den meisten Fällen die Heilstät tenbehandlung - einzuleiten ist.

Das zuverlässige röntgenologische Kontroll- und Ergänzungsmittel jeder genauen Lungenuntersuchung gibt über die Aktivität oder Inaktivität des tuberkulösen Prozesses allein keine sichere Entscheidung. Der einwandfreie bakteriologische Nachweis von Tuberkelbazillen im Auswurf des Kranken ist meist auch bei vollkommenem Mangel jedes anderen pathologisch-nachweisbaren Befundes entscheidend für die weitere Behandlung, sicher aber Beobachtung.

Der noch so oft verwertete, positive Ausfall der Pirquetschen Kutanreaktion ist bezüglich der Diagnose bei Erwachsenen wertlos. Trotzdem sind die gutachtlichen Äusserungen nicht selten, die auf Grund von "Pirquet" positiv in Verbindung mit anderen zufälligen klinischen Erscheinungen die Behandlung des ,Lungenkranken“ für 
dringend notwendig halten, mit anderen Worten, den Antrag auf Durchführung einer Heilstättenkur damit für begründet erachten.

In weit grösserem Masse trifft dies zu, wenn diese Entscheidung dem positiven Ausfall der subkutanen Tuberkulinreaktion überlassen wird, eine Schlušsfolgerung, der man auch bei den oben angeführten Fällen begegnet. Stich- und Fieberreaktion gelten dann für gewöhnlich als ausschlaggebend ohne Rücksicht darauf, dass ersterer durchweg keine grössere Bedeutung zukommt, als der positiven Pirquetschen Reaktion, und dass ferner das Auftreten von Fieber oft genug von den verschiedenartigsten Zufälligkeiten abhängig ist und vor allem auch bei abgeheilten Tuberkulosen nur in den seltensten Fällen fehlt. Welche Bedeutung der Herdreaktion als aktivem Vorgang beizumessen ist, darüber gehen die Ansichten mancher Autoren noch immer auseinander. Sicher st jedenfalls, dass der einwandfreie Nachweis einer Herdreaktion: lokalisierte Brustschmerzen, vermehrter Husten und Auswurf, bazillenhaltiges oder blutiges Sputum, vornehmlich aber der Nachweis frischer oder vermehrter Rasselgeräusche, für das Bestehen eines aktiven Prozesses massgehend sind. $\mathrm{Ob}$ es sich aber in jedem einzelnen Falle nur um ein Deutlicherwerden bestehender aktiver Herderscheinungen handelt, oder ob durch die Tuberkulinreizung ein abgeheilter latenter Herd aus seiner Ruhe gebracht die ersten unbeilvollen Lebenszeichen von sich gibt - die sichere Entscheidung dieser kritischen Frage ist ontscheidend für die Existenzberechtigung des Tuberkulins als znverlässiges und vor allem unschädliches diagnostisches Hilfsmittel. Jedenfalls aber hat Goldscheider (Deutsche med. Wochenschritt $1918,4)$ Recht, wenn er ebenfalls warnend hervorhebt, dass jede Aktivierung eines ruhenden Herdes bereits an sich eine Schädigung bedeutet, von der man nie wissen kann, welchen weiteren Verlauf dieselbe nimmt. Es ist anch kein Grund vorhanden, oder wie Goldscheider sagt, ganz und gar abzulehnen, bei Leuten, die keine Lungenerscheinungen darbieten, solange zu spritzen, bis sich endlich, oft erst nach "sprunghaft gesteigerten Dosen" an der Lunge etwas zeigt. Auch die Anwendung kleinster Dosen schliesst eine Schädigung nicht deshalb aus, weil dieselbe nicht klinisch nachweisbar ist. Und welchen Scbwierigkeiten man bei der richtigen Bewertung des physikalischen Befundes auch bei der grössten Selbstkritik ausgesetzt ist, darüber weiss mancher $z u$ berichten, dem es selbst im geordneten Krankenhausbetrieb trotz wiederholter Untersuchungen schwer fiel, aus dem vermehrten physikalischen Befund den objektiven Nachweis einer sicheren Herdreaktion anzunehmen, wie dies auch Friesecke (Münch. med. Wochenschr. 1917, 46) an der Hand kritisch beleuchteter Einzelheiten richtig hervorhebt. In der Erkenntnis dieser genannten Schwierigkeiten ist es daher eine Selbstverständlichkeit, dass die Tuberkulindiagnostik nur dem Krankenhaus überantwortet bleiben darf.

$\mathrm{Ob}$ derselben in der vorderband wichtigsten Frage, der $\mathrm{Aus}$ w a h'l behandlungsbedürftiger Lungenkranker für die Heilstätten eine ausschlaggebende Bedeutung zukommen wird, muss nach den angedeuteten Ergebnissen mit Recht bezweifelt werden. Vorläufig wird dem praktischen Arzte bei dieser gewaltigen Arbeit die schwerste 
Aufgabe zufallen, wenn er auf Grund seines Befundes nur diejenigen für die bevorzugten Heilstättenplätze aussuchen soll, die nach seinem Ermessen in erster Linie die sicherste Gewähr bieten, nach erfolgter Kur dem wirtschaftlichen und körperlichen Kampfe für immer oder doch auf Jahre hinaus wieder gewachsen zu sein. Diese gewiss noch recht bedeutende Anzahl behandlungsbedürftiger Tuberkulösen würde alsdann bei genauester Berücksichtigung des objektiven Befundes uni der Leistungsfähigkeit unter den uns zu Gebote stehenden therapeutischen Hilfsmitteln mit grösserer Wahrscheinlichkeit unsere Auslagen bezahlt machen wie bisher und dieser gerechten Forderung wird man sich nach dem Kriege am allerwenigsten entziehen kömen.

$\mathrm{Zu}$ diesen erschwerten wissenschaftlichen Forderungen einerseit: tritt noch hinzu der empfindliche Ausfall geübter Arrte und die allgemeine Überlastung. Beide Erscheinungen verdienen zux Zeit die allergrösste Berücksichtigung schon aus dem Grunde, weil sie zuerst als mildernde Umstände für die vielen Fehldiagnosen bei der Lungentuberkulose in Frage kommen. Ganz abgesehen von der Unmöglichkeit einer exakten und ausgiebigen Untersuchung wird dem vielbeschäftigten Arzte auch meist keine Zeit zur l'rüfung wertvoller anannestischer Angaben zur Vertügung stehen. In solchen Fällen ist dann natürlich eine Ergänzung oder Verwertung dieser beiden wichtigen Jomente nicht möglich oder fals:h: denn wenn z. B. in de: Anamnese von häufigem oft sogar sehr starkem Bhuthusten die liede ist, der schon seit Jahren bestancen haben soll und in der biagnose heisst es alsdann "Beginnender Lungenspitzenkatarrh", dann weiss man nicht, ob man jetzt dem Arzt oder dem l'atienten dit' grösste Schuld zuschieben soll. Es darf nicht vergessen werden, dass gerade unter dem Einfluss des Krieges mehr wie je das Gewissens. konto manches "Leichtlungenkranken" nicht unerheblich durch übertriebene anamnestische Angaben beschwert worden ist. Keine Zeit wie die Kriegszeit ist dazu angetan, alle jenen interessanten Kranklieitsbilder zu vervielfältigen, die zum grössten Teil, sei es durch Über- oder Unterschätzung des körperlichen Leidens, sei es durch eine psychische Hemmung, jenen Fällen zugerechnet werden müssen. die Ir öhler (Beitr. z. Klin. d. 'Tuberk. 1907, VIII) unter dem Namen der psychophysischen Gleichgewichtsstörung beobachtet und beschrieben hat. Gerade dem beobachtendeu praktischen Arzte bietet der jetzige Krieg in der Vielgestaltigkeit nie gekannter Verhältnisse die wunderlichsten und oft unmöglichsten Krankbeitsbilder. die auf Grund einer neurasthenischen Veranlagung und lirankhafter Selbsteinschätzung insbesondere bei der. Beurteilung der 'Tuberkulose' selbst den erfahrensten Praktiker in Verlegenheit zu setzen vermögen.

Keine Krankheit wie die Lungentuberkulose bietet so viele und verschiedenartige subjektive und objektive Beschwerden, die jetzt nicht eine annehmbare zeitgemässe Erklärung fünden, keine Krankheit in Wahrheit aber auch eine so grosse Anzahl eigentümlicher Symptome als natürlichste Begleiterscheinungen des Krieges. Auf keinem Gebiet* findet das grosse Heer der Neurastheniker und Simulanten eine solche Answahl passendes und unpassendes Flickzeug anamnestischer Angaben. 
und bei keiner Krankheit endlich gewinnt der Patient und besonders der im Felde gewesene in solchem Masse das natürlichste und verständlichste Entgegenkommen des Arztes. insbesondere wenn diesem noch eine leichtempfängliche Zufriedenheit und weitherzige Rücksichtnalsme eigen ist. Und welche Schwierigkeiten entstehen da nicht fiir den vielbeschäftigten und gewissenhaften Arzt, wenn er bei der objektiven Intersuchung oit allein schon bei der Inspektion des elend aussehenden Patienten seinen Gesamteindruck nicht mit dem oft negativen spezifisch-physikalischen Lungenbefunde in Einklang $z u$ bringen vermag. Unter dem Findruck all dieser Finflüste und nicht zuletyt durch die rielseitige und allgemein gehaltene Fragestellung der (iutacintenformulare lassen sich alsdamn die oft widersprechenden gutachtlichen Iusstrungen in den ..Freifahrtscheinen" fïr Iungenheilstiatten erkliiren.

Die oben angetührten gutachtlichen lintersuchung-befunde. die nur vereinzelt aus einer (iesaintanzahl ron liaum 150 herausgegriffen wurden, sind gewiss besorgliche Tatsachen, an denen die fortschrittlichen Bestuebungen unserer zukiinftigen 'T'uberkulosebekämpfung nicht achtlos und gutmütig vorübergehen dürtell. Bei der grossen Verantwortung alier. die dem praktischen Arzte anf diesem werade indenletztenJahrunsovervollkommnetenGebiete gewissermasich alliredrungen wird. ist es eine l'flicht

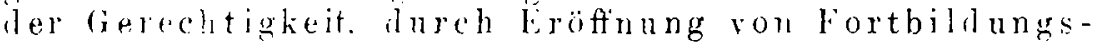
mögliciktiten jeden Arzte delegonhtit zugeben, sein. Wissen und lommen in dieser beziehnng zu bereichern. vornehmlich aber dann. wenn ihm auch wejterhin die verantwortungsvollste und erste Stellung in del liekämptung unserer grössten Volkskraukheit erhalten bleibes soll.

Es ist keine lrage, dass die wissenschattichen und statistischen Erfahrungen Fon Arzten und Versicherungsanstalten schon jetzt die brkemntnis gezeitigt haben, dass auch der Einheitlichkeit, (ienaugkeit und Ubersichtlichkeit der Gutachtentragen eine besondere Anfmerksamkeit nottut, wenn die Gutachten für die Folgezeit als sichere Geleithriefe für die Lungenheilstätton und als wertvolles statistisches Material Verwendung finden sollen. Fs wiirde zu weit führen. an dieser Stelle für diese Forderung schun bestimnte Auhaltspunkte festlegen zu wollen.

In der Hauptsache sollten die gesamten ancreführten Tatsachen und Erfahrungen. die sich in allfallender Weise mit den kürzlich veröffentlichten beachtenswerten Ausfïhrungen von $B$ ït $\operatorname{ta}$ er-W obst Münch. med. Wochenschr. 1918. 6) erfreulicherweise decken, kurz einige besondere Momente berühren, die in bestimmter Weise dazu beigetragen haben, die statistischen Angaben uber die $\Lambda$ usdehnung der Tuberkulose in einem gedämpften aber für uns günstigeren lichte erscheinen zu lassen. Jedenfalls stehen wir vorläutig - unter dem Einfluss des Krieges - den gesteigerten Zahlen über die Ausdehnung der 'Tuberkulose noch mit einem verständlichen l'essimismms gegenïber. 
Nicht wenige, auch namhafte Autoren (de la Camp, Gerhartz, Goldscheider, Hochhaus, Kaiserling, Schröder, Ruppel usw.) haben der günstigen Beeinflussung des Krieges auf die Tubtrkulose das Wort geredet, und manche versuchen auf Grund anderer best mmter Tatsachen anzunehmen, dass die Krankheitsziffer ganz gewiss nicht so gross ist, wie man allgemein annimmt. Es ist nicht zu leugnen, dass für manche der Felddienst unter Stärkung und Kräftigung ibrer Konstitution und Widerstandskraft Besserung und Heilung thres Lungenleidens brachte, dass ferner durch die auffallende und schnelle Zunahme der natürlichen und unnatürlichen Tuberkulosetodesfälle eine erhebliche Abnahmo besonders der Schwerkranken eingetreten ist. Weiter ist nicht von der Hand zu weisen, dass durch die lange Abwesenlıeit vieler schwerkranker Männer für eine grosse Anzahl von Kindern - viele gerade im infektionsfähigsten Alter die sichere Infektionsquelle nicht mehr vorhanden war. Die einwandfreie Nachprüfung dieser wichtigen Tatsache durch Untersuchungen der betreffenden Familien nach dem Kriege wird vielleicht in der richtigen Gegeniiberstellung gewisse Anlıaltspunkte über die Ausdehnung der Kindertuberkulose geben, eine Feststellung, die überhaupt für die Ausdebnung der Tuberkulose im allgemeinen als sicherstes Ergebnis allsschlaggebend ist. Ferner wird es an der Hand umfangreicher militärärztlicher Aufreichnungen durch genaueste Kontrollund Naclıuntersuchungen möglich sein, über die Ausdehnung der Tuberkulose bei der männlichen Bevölkerung andere, günstigere statistische /ahlenverhältnisse zu gewinnen, die in den angefuhrten Boob. achtungen und Erfahrungen eine natürliche und erireuliche Erklärung finden.

Nur unter Ausnutzung dieser und anderer wertwoller Kriegserfahrungen und bei weitg.hendster Inanspruchnahme fachärztlich geschulter und erfahrener Ärıte werden wir mit Erfolg den bevorstehenden Kampf gegen die aktive Tuberkulose aufnehmen können.

Insbesondere muss, als erster Grundpieiler, die ärztliche Auswahl der Kranken für die Volksheilstätten auf eine wissenschaftlich einwandfreie Beurteilung Anspruch machen können, nachdem uns der Krieg mit zwingender Notwendigkeit über die Platzfrage in Lungenheilstätten eines anderen belehrt hat. is darf fortan nicht mehr der allıusehr herausgekehrte Standpunkt vorberrschen, die Schwerkranken aus den Heilstätten fernzuhalten. sondern vielmehr alle diejenigen, bei denen nicht mit den üblichen diagnostischen Hilfsmitteln mit Sicherbeit eine aktive Tuberkulose festgestellt werden kann. Sicherlich werden dann die statistischen Erfolge unserer Heilstätten ein andores, allerdings weniger erfreuliches, Bild geben, aber die wirklichen Erfolge werden, wie Büttner-Wobst (1. c.) ebenfalls mit Recht betont, in den Heilstätten mit der scblechtesten Statistik zu finden sein, weil dort die beste und zielbewussteste Arbeit geleistet wird. Auf diese Weise würde auch die Existenzfähigkeit mancher Heilstätten auf gesunderer Basis begründet sein und manchen Heilstättenarzt einer verständlichen Weitherzigkeit überheben, zu der er oft genug im Interesse einer gesunden Finanzierung seiner Anstalt notgedrungen gezwungen ist. Die Einrichtung und Erweiterung fach- 
17] Irrungen und Wirrungen in dem Kampfe gegen die Tuberkulose. 169

ärztlich geleiteter Beobachtung s s tationen (Vor- und Durchgangsstationen, wie sie vor dem Kriege bereits von 17 Versicherungsanstalten mit bestem Erfolg eingerichtet wurden, wïrden alsdann neben ihrer allgemein anerkannten notwendigen Zweckmässigkeit nicht allein die sicherste Gewähr für eine geeignete Auswahl unserer Volksheilstätten bieten, sondern auch in ihrem wissenschaftlichen Ausbau allmählich zu massgebenden Forschungs- und Bildungsstätten heran. wachsen, wie sie unserer ausgedehntesten, bösartigsten und doch so vielfach heilbaren chronischen Erkrankung - der Tuberkulose zukommen. 